\title{
Pseudomonas aeruginosa infection and exacerbations in bronchiectasis: more questions than answers
}

\author{
Miguel Angel Martinez-García \\ Affiliation: Hospital Universitario y Politécnico la Fe de Valencia, Valencia, Spain.
}

Correspondence: Miguel Angel Martinez-García, Hospital Universitario y Politécnico la Fe de Valencia, Pneumology Service, Bulevar Sur s/n Valencia, Valencia 46013, Spain.

E-mail: mianmartinezgarcialagmail.com

@ERSpublications

Both chronic infection by $P$. aeruginosa and exacerbations have a negative impact in bronchiectasis prognosis http://ow.ly/pjR230hJR9h

Cite this article as: Martinez-García MA. Pseudomonas aeruginosa infection and exacerbations in bronchiectasis: more questions than answers. Eur Respir J 2018; 51: 1702497 [https://doi.org/10.1183/ 13993003.02497-2017].

Apart from the presence of comorbidities [1], there are two other circumstances that have demonstrated a negative impact on the natural history of bronchiectasis: chronic bronchial infection (CBI) by Pseudomonas aeruginosa and exacerbations. Many studies have shown an association between CBI by $P$. aeruginosa and reduced quality of life [2], a steep decline in lung function [3], increased inflammation (both bronchial and systemic) [4], higher healthcare costs [5], and even a higher mortality rate [6]. One recent meta-analysis concluded that this association is more evident with CBI by $P$. aeruginosa rather than by other pathogenic microorganisms [7]. Similarly, exacerbations (particularly multiple and/or severe exacerbations) have been associated with various parameters of severity in bronchiectasis [3], including a higher mortality rate $[8,9]$. It is hardly surprising, therefore, that the three validated multidimensional scores published to date to evaluate the severity or prognosis of bronchiectasis [8-10] include the presence of a CBI by $P$. aeruginosa as one of their variables, and that two of the three $[8,9]$ also include the frequency and/or severity of exacerbations. Accordingly, some authors have even suggested that those bronchiectasis patients with CBI by $P$. aeruginosa and those who present multiple exacerbations could represent two distinct clinical phenotypes of bronchiectasis patients, each with their own clinical characteristics and prognoses [11, 12]. Finally, it should be stressed that both these circumstances are extremely important from a therapeutic standpoint. In fact, the vast majority of the treatments on the market or in development are primarily aimed at infection by $P$. aeruginosa, with a drop in the number of exacerbations as the main objective [13].

Nevertheless, despite recent advances in our knowledge of both acute and chronic infection in bronchiectasis, many questions remain unanswered, and some of these have a particular clinical significance. It is well known that patients with CBI by $P$. aeruginosa present more, and more severe, exacerbations [14], and so it is pertinent to ask: Is the relationship between the presence of a CBI by $P$. aeruginosa and a poorer prognosis of bronchiectasis necessarily mediated by the presence of exacerbations? Or, in other words: Do those patients with CBI by $P$. aeruginosa who present no exacerbations, or only a few, also have a poorer prognosis? The answer to this question is crucial to any real understanding of the disease's natural history, and it could also have a profound therapeutic impact.

Received: Dec 022017 | Accepted: Dec 042017

Conflict of interest: None declared.

Copyright OERS 2018 
In the current issue of the European Respiratory Journal, Araújo et al. [15] try to provide an answer to this question, using a large multinational cohort of bronchiectasis patients with different baseline characteristics. These authors use a multivariate analysis to establish the relative independent risk of mortality in four groups of patients assembled on the basis of combinations of two variables: the presence or absence of CBI by $P$. aeruginosa and the presence or absence of at least two exacerbations per year. The authors conclude that, although the presence of a CBI by $P$. aeruginosa was generally associated with a lower quality of life, it was only associated with higher mortality in those groups with multiple (at least two) annual exacerbations, and this was especially the case when they also presented CBI by P. aeruginosa. These results extend the scientific evidence on the enormous importance of both preventive treatment of exacerbations and treatment of exacerbations themselves (regardless of the microorganism that caused them), and they endorse the general approach recommended by the recently published European bronchiectasis guidelines [16] of using long-term inhaled antibiotics to treat those patients with CBI by $P$. aeruginosa and multiple exacerbations (at least three per year according to these guidelines). However, as so often occurs in science, this study also throws up many other extremely interesting questions. Perhaps one of the most important clinical questions is whether patients with CBI by $P$. aeruginosa but few exacerbations should be treated with long-term (preferably) inhaled antibiotics, although this situation is much less common in clinical practice (ARAújo et al. [15] found only $15.9 \%$ of patients with CBI by $P$. aeruginosa and fewer than two exacerbations per year, and this percentage is similar to those found in other large databases $[9,10])$.

There are many arguments for and against taking a therapeutic decision along these lines as have been stated by some authors and different guidelines [17]. Although it is true that there does not seem to be any excess mortality in the absence of exacerbations, we do know that the presence of a CBI by $P$. aeruginosa induces a deterioration in a patient's quality of life (as evidenced in the study by ARAújo et al. [15], and in others in the scientific literature [2, 15]), and a greater clinical severity (the percentage of asymptomatic patients with CBI by P. aeruginosa is extremely low) [8-10]. Both these factors could be improved by treating the CBI, as has been demonstrated by several clinical trials [18, 19]. Nevertheless, although some clinical trials with inhaled antibiotics have included patients with multiple exacerbations $[20,21]$ (while some, such as the one on inhaled colistin [19], did not require any previous exacerbations at all), these exacerbations were only recorded in the year prior to inclusion which does not mean the same future yearly average of exacerbations. Furthermore, we still do not know whether the presence of a CBI by $P$. aeruginosa is merely a marker of the severity of bronchiectasis or whether it is the cause of subsequent multiple exacerbations $[22,23]$ and whether, therefore, its treatment could prevent the onset of these exacerbations and thereby improve the prognosis. We also come across fragile patients affected by CBI by $P$. aeruginosa and by several comorbidities (of a cardiorespiratory nature, for example) who have few exacerbations, but these exacerbations are very severe due to the patients' deteriorated baseline situation.

On the other hand, it is impossible to ignore the arguments against long-term treatment with inhaled antibiotics. More particularly, there are no specific studies of patients with CBI by $P$. aeruginosa and few exacerbations that clearly establish any indication for their treatment and, anyway, long-term treatment often has adverse effects (generally of a local nature). Such treatment also represents a considerable financial burden for the management of bronchiectasis patients [5] and this is difficult for many healthcare systems to assume.

Maybe we can take a more individualised therapeutic approach to patients with CBI by $P$. aeruginosa, as the decisions concerning this subgroup of patients largely depend on their personal characteristics, the accessibility and cost of the medication, as well as the microbiological environment of their home country with respect to the prevalence of CBI by $P$. aeruginosa and the pattern of resistance existing there (as in the case of most infectious diseases). Maybe additional data for specific geographical areas could be taken from national guidelines on bronchiectasis (probably this is why national guidelines are also important) where the recommended general treatment of international guidelines could be sometimes modified according to particular local circumstances [24]. The study by ARAújo et al. [15] for example, shows how the prevalence of CBI by $P$. aeruginosa varies enormously from one country to another, ranging from $0.9 \%$ in Serbia and $12.5 \%$ in the UK to over $21 \%$ in Spain and around $40 \%$ in Greece and Israel; and the latter percentage may possibly be matched, or even surpassed, in Latin American countries [25] and China [26]. It may therefore be appropriate to start treatment with inhaled antibiotics in patients with marked clinical deterioration as a result of CBI by $P$. aeruginosa; or in those with several comorbidities (particularly in countries with an ageing population that is therefore subject to a higher probability of concomitant diseases); or in microbiological environments with a high prevalence of CBI by $P$. aeruginosa or a high level of resistance to treatment, to avoid any deterioration in the microbiological situation (many of the multi-resistant $P$. aeruginosa maintain a good sensitivity to colistin, a drug available via the inhaled route [27]). Inhaled antibiotics are particularly suited to countries where they are not too difficult or expensive 
to acquire and where there is already a substantial past history of their administration. And while we await new evidence on both the most appropriate type of patient for inhaled antibiotic treatment and its cost-effectiveness, concerted efforts are being made internationally to unify definitions (of, for example, exacerbation and chronic bronchial infection) and establish the clinical and radiological criteria for diagnosis that should be used in future clinical studies [28]. Such a unification would represent an incalculable advance in terms of scientific rigour. Furthermore, long-term longitudinal studies based on national and international registries, which are currently mushrooming at an extraordinary rate, could provide answers to the many questions that are still unresolved. Such studies will help us trace the natural history of bronchiectasis and establish the chronological order of the disease's pathological events. This will bring us closer to a preventive medicine that could anticipate such events (for example, CBI by $P$. aeruginosa and exacerbations).

We also need studies with biomarkers [29] that could indicate which patients with pre-established CBI by $P$. aeruginosa will present a better or worse prognosis or a higher or lower number of exacerbations, as this advance would enable us to offer our patients more personalised treatment. There is also a need for clinical studies on the cost-effectiveness of treatment with long-term inhaled antibiotics specifically designed for patients with CBI by $P$. aeruginosa and few exacerbations, using important clinical outcomes other than exacerbations (for example, clinical changes or alterations to the quality of life).

While we await this type of new evidence, excellent studies like that of Araújo et al. [15] are very welcome, as they increase the scientific evidence on a subject of the utmost clinical importance and they make clear, yet again, the need to continue investigating the impact of $P$. aeruginosa and exacerbations on the natural history of bronchiectasis, in order to extend our knowledge of these matters to a point where we will have more answers than questions.

\section{References}

1 McDonnell MJ, Aliberti S, Goemminne PC, et al. Comorbidities and the risk of mortality in patients with bronchiectasis: an international multicentre cohort study. Lancet Respir Med 2016; 4: 969-979.

2 Wilson CB, Jones PW, O'Leary CJ, et al. Effect of sputum bacteriology on the quality of life of patients with bronchiectasis. Eur Respir J 1997; 10: 1754-1760.

3 Martinez-Garcia MA, Soler-Cataluna JJ, Perpina-Tordera M, et al. Factors associated with lung function decline in adult patients with stable non-cystic fibrosis bronchiectasis. Chest 2007; 132: 1565-1572.

4 Chalmers JD, Smith MP, McHugh BJ, et al. Short- and long-term antibiotic treatment reduces airway and systemic inflammation in non-cystic fibrosis bronchiectasis. Am J Respir Crit Care Med 2012; 186: 657-665.

5 de la Rosa D, Martinez-Garcia MA, Olveira C, et al. Annual direct medical costs of bronchiectasis treatment: impact of severity, exacerbations, chronic bronchial colonization and chronic obstructive pulmonary disease coexistence. Chron Respir Dis 2016; 13: 361-371.

6 Loebinger MR, Wells AU, Hansell DM, et al. Mortlaity in bronchiectasis: a long-term study assessing the factors influencing survivial. Eur Respir J 2009; 34: 843-849.

7 Finch S, McDonnell MJ, Abo-Leyah H, et al. A comprehensive analysis of the impact of Pseudomonas aeruginosa colonization on prognosis in adult bronchietasis. Ann Am Thorac Soc 2015; 12: 1602-1611.

8 Chalmers JD, Goeminne P, Aliberti S, et al. The Bronchiectasis Severity Index: an international derivation and validation study. Am J Respir Crit Care Med 2014; 189: 576-585.

9 Martinez-Garcia MA, Athanazio RA, Giron R, et al. Predicting high risk of exacerbations in bronchiectasis: The E-FACED score. Int J Chron Obstruct Pulmon Dise 2017; 18: 275-284.

10 Martinez-Garcia MA, de Gracia J, Vendrell Relat M, et al. Multidimensional approach to non-cystic fibrosis bronchiectasis: the FACED score. Eur Respir J 2014; 43: 1357-1367.

11 Aliberti S, Lonni S, Dore S, et al. Clinical phenotypes in adult patients with bronchiectasis. Eur Respir J 2016; 47: $1113-1122$.

12 Martinez-Garcia MA, Vendrell M, Giron R, et al. The multiple faces of non-cystic fibrosis bronchiectasis. A cluster analysis approach. Ann Am Thorac Soc 2016; 13: 1468-1475.

13 Chalmers JD, Loebinger M, Aliberti S. Challenges in the development of new therapies for bronchiectasis. Expert Opin Pharmacother 2015; 16: 833-850.

14 Chawla K, Vishwanath S, Manu MK, et al. Influence of Pseudomonas aeruginosa on exacerbation in patients with bronchiectasis. J Glob Infect Dis 2015; 7: 18-22.

15 Araújo D, Shteinberg M, Aliberti S, et al. The independent contribution of Pseudomonas aeruginosa infection to long-term clinical outcomes in bronchiectasis. Eur Respir J 2018; 51: 1701953.

16 Polverino E, Goeminne PC, McDonnell MJ, et al. European Respiratory Society guidelines for the management of adult bronchiectasis. Eur Respir J 2017; 50: 1700629.

17 Wilson R, Aksamit T, Aliberti S, et al. Challenges in managing Pseudomonas aeruginosa in non-cystic fibrosis bronchiectasis. Respir Med 2016; 117: 179-189.

18 Murray MP, Govan JR, Doherty CJ, et al. A randomized controlled trial of nebulized gentamicin in non-cystic fibrosis bronchiectasis. Am J Respir Crit Care Med 2011; 183: 491-499.

19 Haworth CS, Foweraker JE, Wilkinson P, et al. Inhaled colistin in patients with bronchiectasis and chronic Pseudomonas aeruginosa infection. Am J Respir Crit Care Med 2014; 189: 975-982.

20 Aksamit T, Bandel TJ, Criollo $\mathrm{M}$, et al. The RESPIRE trials: Two phase III, randomized, multicentre, placebo-controlled trials of Ciprofloxacin Dry Powder for Inhalation (Ciprofloxacin DPI) in non-cystic fibrosis bronchiectasis. Contemp Clin Trials 2017; 58: 78-85.

21 Serisier DJ, Bilton D, De Soyza A, et al. Inhaled, dual release liposomal ciprofloxacin in non-cystic fibrosis bronchiectasis (ORBIT-2): a randomised, double-blind, placebo-controlled trial. Thorax 2013; 68: 812-817. 
22 Evans SA, Turner SM, Bosch BJ, et al. Lung function in bronchiectasis: the influence of Pseudomonas aeruginosa. Eur Respir J 1996; 9: 1601-1604.

23 Davies G, Wells AU, Doffman S, et al. The effect of Pseudomonas aeruginosa on pulmonary function in patients with bronchiectasis. Eur Respir J 2006; 28: 974-979.

24 Martinez-Garcia MA, Máiz L, Olveira C, et al. Spanish guidelines on treatment of bronchiectasis in adults. Arch Bronconeumol 2017; in press [https://doi.org/10.1016/j.arbres.2017.07.016].

25 Athanazio R, Pereira MC, Gramblicka G, et al. Latin America validation of FACED score in patients with bronchiectasis: an analysis of six cohorts. BMC Pulm Med 2017; 17: 73.

26 Lin JL, Xu JF, Qu JM. Bronchiectasis in China. Ann Am Thorac Soc 2016; 13: 609-616.

27 Antoniu SA, Cojocaru I. Inhaled colistin for lower respiratory tract infection. Expert Opin Drug Deliv 2012; 9: 333-342.

28 Hill AT, Haworth CS, Aliberti S, et al. Pulmonary exacerbation in adults with bronchiectasis: a consensus definition for clinical research. Eur Respir J 2017; 49: 1700051.

29 Chalmers JD, Moffitt KL, Suarez-Cuartin G, et al. Neutrophil elastase activity is associated with exacerbations and lung function decline in bronchiectasis. Am J Respir Crit Care Med 2017; 195: 1384-1393. 